\title{
Estructura socioeconómica y hábitos alimentarios en el estado nutricional de los estudiantes del sur peruano
}

\section{Socioeconomic structure and eating habits in the nutritional status of students from southern Peru}

\author{
Luzbeth Lipa Tudela ${ }^{1, a}$ \\ https://orcid.org/0000-0001-7795-1777 \\ Yessica Quilca Soto ${ }^{1, a}$ \\ https://orcid.org/0000-0001-6200-6828
}

Patricia Geldrech Sanchez 1,b

https://orcid.org/0000-0002-2482-4069

\author{
Humberto Mamani-Coaquira 1,c \\ https://orcid.org/0000-0002-0569-860X \\ Jesús Wiliam Huanca-Arohuanca 2,d \\ https://orcid.org/0000-0002-7353-1166
}

\section{Citar como}

Lipa, L., Geldrech, P., Quilca, Y., Mamani-Coaquira, H. y Huanca-Arohuanca, J. W. (2021). Estructura socioeconómica y hábitos alimentarios en el estado nutricional de los estudiantes del sur peruano. Desafíos, 12(2); 133-41.

\section{RESUMEN}

Objetivo. Determinar la relación entre el nivel socioeconómico y los hábitos alimentarios con el estado nutricional de los estudiantes del sur peruano. Métodos. Fue un estudio tipo transversal-descriptiva-analítica. La población estuvo conformada por 1109 adolescentes, con una muestra de 286 escolares del nivel secundario. La recolección de información se efectuó bajo la tutela de la antropometría y la encuesta en función del nivel socioeconómico de las necesidades básicas y los hábitos alimentarios. Resultados. Los resultados develaron que el estado nutricional, según el índice de masa corporal (IMC) total fue de $77,27 \%$, con un estado nutricional normal de $15,73 \%$ en sobrepeso, 4,55\% con obesidad, $1,75 \%$ de riesgo hacia la delgadez y 0,70\% con delgadez; por otro lado, el nivel socioeconómico medio fue $71,73 \%$, el bajo superior de $23,78 \%$, el bajo inferior de 0,35 \% y por último se observó que el hábito alimentario de los estudiantes se encontraba en 1,40 \% para la categoría "muy bueno", 72,38 \% en "bueno", 4,20 \% en "regular", $17,13 \%$ en "malo" y 4,90 \% como "muy malo". Conclusiones. Se concluye que no existe relación entre el nivel socioeconómico y el estado nutricional, pero sí existe una relación entre los hábitos alimentarios y el estado nutricional de estudiantes de la región de los Andes australes del Perú.

Palabras clave: educación; hábitos alimentarios; estudiantes; economía; estado nutricional.

\begin{abstract}
Objective. To determine the relationship between the socioeconomic level and eating habits with the nutritional status of students in southern Peru. Methods. It was a cross-sectional-descriptive-analytical type of study. The population consisted of 1109 adolescents, with a sample of 286 secondary school students. The collection of information was carried out under the supervision of anthropometry and the survey according to the socio-economic level of basic needs and eating habits. Results. The results revealed that the nutritional status, according to the total body mass index (BMI) was $77.27 \%$ with a normal nutritional status of $15.73 \%$ overweight, $4.55 \%$ obesity, $1.75 \%$ risk to thinness and $0.70 \%$ thinness; on the other hand, the average socioeconomic level was $71.73 \%$, the higher low of $23.78 \%$, the lower low of $0.35 \%$, and finally it was observed that students' eating habits were $1.40 \%$ for the "very good" category, $72.38 \%$ for "good", 4.20\% for "regular", 17.13\% for "bad" and 4.90\% as "very bad". Conclusions. It is concluded that there is no relationship between dietary habits and nutritional status of students from the southern Andes region of Peru. Keywords: education; eating habits; students; economy; nutritional status.
\end{abstract}

\section{Filiación y grado académico}

1 Universidad Nacional del Altiplano, Puno, Perú

2 Universidad Nacional de San Agustín de Arequipa, Perú.

\author{
a Doctora en Ciencias de la Salud. \\ b Doctora en Ciencias de la Educación. \\ Magister en Educación. \\ d Magister en Filosofía con mención en Ética y Filosofía \\ Política.
}




\section{INTRODUCCIÓN}

En las últimas décadas, el estado nutricional ha cambiado relativamente, de manera que los jóvenes y adolescentes del ayer son muy diferentes a los de hoy, ya que le dan un valor muy importante a su entorno social, a la forma como se manifiestan frente al colectivo y frente al grupo en el que se desenvuelven. Las adolescentes y jóvenes, por ejemplo, se consideran bellas o no, de acuerdo a su grado de delgadez (Morales-Ruán et al., 2018; Morgante y Remorini, 2018). Por ello, diversas colocaciones relacionadas con la salud, establecen parámetros de pesos adecuados relacionados a las menores tasas de morbimortalidad (Arnaud et al., 2005). Es más, según el Censo de Población y Vivienda del 2007, se registró una población de 12 a 19 años de edad compuesta por 233393 personas, representando el 18,4\% de la población altiplánica (Instituto Nacional de Estadística e Informática, 2007) que presentaba un estado nutricional negativo para su salud.

Otro factor muy relevante que repercute directamente al estado nutricional es el nivel socioeconómico. En este sentido, se han encontrado diferentes estudios que muestran estados nutricionales semejantes en individuos de diferentes niveles socioeconómicos, que presentan un mal hábito alimentario porque carecen de la economía y por lo tanto su alimentación es pésima. De igual manera, los casos de sobrepeso y obesidad cada vez van en aumento, de modo que ya no es problema de los países desarrollados como se creía anteriormente (Uribe-Carvajal et al., 2018), sino que, ahora, es un común denominador de los países periféricos como el Perú, recientemente arrasado por la pandemia (HuancaArohuanca, 2020; Huanca-Arohuanca y Núñez, 2020).

Por otro lado, los hábitos alimentarios también inciden en el estado nutricional de los estudiantes altiplánicos; por decir, si hay un buen hábito alimentario se mantendrá una buena salud (Bobroff, 2012). Es más, el hábito positivo de alimentación puesto en práctica, tendrá como efecto una buena conducta alimentaria (Arnaud et al., 2005), que pueda contrarrestar la desnutrición crónica que a menudo se presenta en el Perú. Según el área de residencia, la desnutrición crónica afectó en mayor proporción a niñas y niños del área rural (26,5\%), es decir, 18,6 puntos porcentuales más que en el área urbana (7,9\%) (Burstein-Roda, 2018); lo cual es un indicador que pone de manifiesto las precariedades existentes en el país.

Bajo estas premisas, el estudio tiene como objetivo determinar la relación entre el nivel socioeconómico y los hábitos alimentarios con el estado nutricional de los estudiantes del altiplano y mostrar, paralelamente, algunos resultados sobre el IMC, el sobrepeso, la obesidad y el riesgo hacia la delgadez. Todos ellos, relacionados a los hábitos alimentarios determinados en una jerarquización manejada en el desarrollo de la investigación.

\section{MÉTODOS}

\section{Tipo de estudio}

La investigación se basó en el diseño cuantitativo, de tipo transversal de carácter descriptivo-analítico (Huanca-Arohuanca et al., 2020; Huanca-Arohuanca y Geldrech, 2020; Huanca-Arohuanca y Pilco, 2021; Huanca-Arohuanca, Supo-Condori, et al., 2020; MiguelAguilar et al., 2017).

\section{Población y muestra}

La población del estudio estuvo constituida por 1109 adolescentes de diferentes colegios secundarios del altiplano peruano, donde se recurrió al muestreo probabilístico aleatorio para obtener una muestra de 286 escolares con un nivel de confianza de 0,95 y un criterio selectivo que consideró a cuarto y quinto grado, tal como se explica en la tabla 1.

\section{Instrumentos y técnicas de recolección de datos}

Se debe precisar que los instrumentos de investigación utilizados en el manuscrito fueron: el cuestionario, la balanza digital, el tallímetro de madera, la ficha

Tabla 1

Población y muestra de los estudiantes altiplánicos

\begin{tabular}{lccc}
\hline Instituciones educativas secundarias & Contexto & Cantidad & Grados \\
\hline María Auxiliadora & Urbano & 50 & Cuarto \\
José Antonio Encinas & Urbano & 40 & Cuarto \\
Gran Unidad Escolar San Carlos & Urbano & 80 & Cuarto \\
José Carlos Mariátegui & Urbano & 40 & Quinto \\
Técnico Industrial & Urbano & 50 & Quinto \\
Nuestra Señora del Carmen & Urbano & 26 & 6 \\
Total & 6 & 286 & \\
\hline
\end{tabular}

Nota: Tomado del registro auxiliar de los docentes. 
de evaluación nutricional y la encuesta de nivel socioeconómico; mientras que la técnica utilizada fue la encuesta de hábitos alimentarios y las fotografías del proceso de recolección de información. Todos ellos han sido elementales para la construcción y recolección de la información.

\section{Procedimiento de la recolección de datos}

El estudio desarrolló tres variables fundamentales que fueron analizadas de manera secuencial. En primera instancia, se abordó el nivel socioeconómico a través de una encuesta de 16 preguntas; así mismo, fueron aplicados puntajes para cada pregunta de acuerdo a la respuesta y fueron sumados los puntos de cada pregunta para generar el resultado final, categorizándose de la siguiente manera: alto, medio, bajo superior, bajo inferior y marginal.

En segunda instancia, fueron analizados los hábitos alimentarios bajo el criterio de la encuesta con 21 preguntas aplicadas a los estudiantes en estudio, categorizándose de la siguiente manera: muy bueno, bueno, regular, malo y muy malo. Es más, la entrevista fue realizada una sola vez, con la finalidad de identificar los hábitos alimentarios de los estudiantes en estudio.

En un tercer momento, se trabajó el estado nutricional bajo los estándares del método antropométrico (NavarroAburto et al., 2017), tomando como indicador el IMC, el cual se relaciona con el peso cuadrado de la talla (peso/ $\mathrm{cm}^{2}$ ) de todos los estudiantes correspondientes a diversos colegios del altiplano austral del Perú.

\section{Análisis de datos}

Los resultados obtenidos fueron sometidos a la prueba estadística chi-cuadrada para determinar si existe relación entre el nivel socioeconómico y los hábitos alimentarios con el estado nutricional de estudiantes del sur peruano.

\section{Aspectos éticos}

Cumpliendo la normativa de las instituciones del sur peruano, se tuvo un aval de los directores de las entidades ya mencionadas de manera precedente. Cabe señalar que la institución que autorizó previo a un dictamen ético fue la Facultad de Nutrición Humana de la Universidad Nacional del Altiplano.

\section{RESULTADOS}

\section{El estado nutricional de los escolares del sur peruano}

El estado nutricional de los estudiantes tomó como indicador el IMC. Por ello, los resultados obtenidos en la tabla 2 representaron a toda la muestra de estudiantes conformada por un número de 286, de la cual, el 77,27\% se encontró con un estado nutricional normal, el 15,73 \% se encontró con sobrepeso, el 4,55\% estuvieron con obesidad, el 1,75\% presentó riesgo de delgadez y el 0,70\% tenía delgadez.

De tal manera, los estudiantes que presentan bajo peso en el estudio descuidan su dinámica de alimentación, ya sea por la presión de las labores escolares, el estrés añadido o por los malos hábitos que traen consigo. Por otra parte, los que tienen sobrepeso rompen las reglas de su alimentación por consumir comida rápida empaquetada, como son: las papitas saladas, biscochos, pastelillos, chizitos, galletas rellenas con crema, gaseosas y chocolates. Todos ellos calman el hambre pero no aportan el valor nutritivo suficiente que el organismo necesita.

Además, cuando se verifica la disponibilidad de productos y alimentos expendidos en las tiendas escolares, se hace notorio que aquellos productos no ofrecen una comida balanceada. De tal manera que, para cubrir las necesidades nutricionales de los estudiantes, las tiendas escolares deberían proveer productos con contenido de alto grado en caloría y proteínas a sus principales consumidores. Por consiguiente, mantener una buena salud dependerá de una actividad física basada en ejercicios que ayudan a mantener el peso corporal dentro de los rangos de normalidad y mantenerse clínicamente sanos.

\section{Nivel socioeconómico de las familias}

En el apartado se visualiza que la mayoría de familias de los escolares del sur peruano se ubicaron entre el apartado medio y bajo-superior. Lo cual indica una condición socioeconómica preocupante tanto para la institución y para la familia como residencia concreta. En efecto, el nivel socioeconómico medio estuvo en un $71,73 \%$, mientras que el nivel bajo superior concentró un $23,78 \%$, seguido del nivel bajo inferior con un $0,35 \%$ y solo el 4,55\% pertenecían al nivel socioeconómico alto.

De lo anterior, se extrae un nivel socioeconómico alto del 4,55\%; situación que representa a estudiantes con mayor acceso a los servicios superfluos y que pueden acceder a una alimentación variada, puesto

Tabla 2

Estado nutricional de los estudiantes del sur peruano

\begin{tabular}{lcc}
\hline Estado nutricional & fi & $\%$ \\
\hline Delgadez & 2 & 0,70 \\
Riesgo de delgadez & 5 & 1,75 \\
Normal & 221 & 77,27 \\
sobrepeso & 45 & 15,73 \\
Obesidad & 13 & 4,55 \\
Total & 286 & 100 \\
\hline
\end{tabular}

Fuente. ficha de evaluación nutricional. 
Tabla 3

Nivel socioeconómico de las familias de los escolares del sur peruano

\begin{tabular}{|c|c|c|c|c|c|c|c|c|c|c|c|c|}
\hline \multirow{3}{*}{ Nivel Socioeconómico } & \multicolumn{10}{|c|}{ IMC } & \multirow{2}{*}{\multicolumn{2}{|c|}{ Total }} \\
\hline & \multicolumn{2}{|c|}{ Delgadez } & \multicolumn{2}{|c|}{$\begin{array}{l}\text { Riesgo de } \\
\text { delgadez }\end{array}$} & \multicolumn{2}{|c|}{ Normal } & \multicolumn{2}{|c|}{ Sobrepeso } & \multicolumn{2}{|c|}{ Obesidad } & & \\
\hline & $\mathrm{fi}$ & $\%$ & $\mathrm{fi}$ & $\%$ & $\mathrm{fi}$ & $\%$ & $\mathrm{fi}$ & $\%$ & $\mathrm{fi}$ & $\%$ & $\mathrm{fi}$ & $\%$ \\
\hline Alto & 0 & 0,0 & 0 & 0,0 & 10 & 3,5 & 3 & 1,0 & 0 & 0,0 & 13 & 4,5 \\
\hline Medio & 1 & 0,3 & 5 & 1,7 & 160 & 55,9 & 30 & 10,5 & 8 & 2,8 & 204 & 71,3 \\
\hline Bajo Superior & 1 & 0,3 & 0 & 0,0 & 51 & 17,8 & 11 & 3,8 & 5 & 1,7 & 68 & 23,8 \\
\hline Bajo Inferior & 0 & 0,0 & 0 & 0,0 & 0 & 0,0 & 1 & 0,3 & 0 & 0,0 & 1 & 0,3 \\
\hline Total & 2 & 0,7 & 5 & 1,7 & 221 & 77,3 & 45 & 15,7 & 13 & 4,5 & 286 & 100 \\
\hline
\end{tabular}

Fuente. ficha del nivel socioeconómico e IMC.

que la selección de los alimentos dependerá mucho tanto sobre el conocimiento sobre nutrición de los padres familia, como el de ellos mismos. No obstante, la mayoría de la población aun no encuentra esa variabilidad para presuponer una mejor alimentación. Concretizando así, una diferencia de clases muy notorio entre estudiantes de la zona urbana y la esfera rural con carencias interminables.

\section{Una mirada a los hábitos alimentarios}

El hábito, como generalidad, representa constancia y determinación, más aún cuando a alimentación se refiere. En el artículo se observa que los estudiantes tienen, en su mayoría, hábitos alimentarios que están en la categoría de muy bueno y bueno, representados en $1,40 \%$ y $72,38 \%$, respectivamente. Sin embargo, la preocupación de los actores educativos está centrada en paliar la cifra regular del 4,20\%, en eliminar el nivel malo de $17,13 \%$ y erradicar con urgencia el nivel muy malo representado en un 4,90\%, tal como se precisa en la tabla 4.

Los hábitos alimentarios son patrones y conductas alimentarias implantadas a lo largo de la vivencia familiar, influenciados por múltiples factores como: medios de comunicación, industria alimentaria, entorno social y factor económico. Los hábitos alimentarios de los estudiantes son, en general, distintos a los adultos, ya que tienden a saltarse comidas, es decir, comen la mayoría de veces fuera de sus hogares y consumen refrigerios, gaseosas, golosinas y comida rápida. De tal modo, estos han incrementado el consumo de alimentos industrializados con alto valor calórico y casi nulo valor nutricional.

A lo anterior, se agrega que los hábitos alimentarios en la etapa estudiantil se ven influenciados por factores cotidianos como el trabajo de los padres, el apoyo familiar a los abuelos, las tendencias a los centros escolares, los recursos económicos bien diferenciados entre pobres y ricos que comparten el mismo colegio.

\section{Comprobación de la primerahipótesis específica del nivel socioeconómico}

Ha: Existe relación entre los hábitos alimentarios con el estado nutricional de escolares del sur peruano.

Ho: No existe relación entre el nivel socioeconómico y el estado nutricional de escolares del sur peruano.

Tabla 4

Hábitos alimentarios de los escolares del sur peruano

\begin{tabular}{|c|c|c|c|c|c|c|c|c|c|c|c|c|}
\hline \multirow{3}{*}{ Hábitos alimentarios } & \multicolumn{10}{|c|}{ IMC } & & \\
\hline & \multicolumn{2}{|c|}{ Delgadez } & \multicolumn{2}{|c|}{$\begin{array}{l}\text { Riesgo de } \\
\text { delgadez }\end{array}$} & \multicolumn{2}{|c|}{ Normal } & \multicolumn{2}{|c|}{ Sobrepeso } & \multicolumn{2}{|c|}{ Obesidad } & \multicolumn{2}{|c|}{ Total } \\
\hline & $\mathrm{N}^{\circ}$ & $\%$ & $\mathrm{~N} .^{\circ}$ & $\%$ & $\mathrm{~N} .^{\circ}$ & $\%$ & $\mathrm{~N} .^{\circ}$ & $\%$ & $\mathrm{~N} .^{\circ}$ & $\%$ & $\mathrm{~N} .^{\circ}$ & $\%$ \\
\hline Muy bueno & 0 & 0,0 & 0 & 0,0 & 4 & 1,4 & 0 & 0,0 & 0 & 0,0 & 4 & 1,4 \\
\hline Bueno & 0 & 0,0 & 0 & 0,0 & 207 & 72,4 & 0 & 0,0 & 0 & 0,0 & 207 & 72,4 \\
\hline Regular & 0 & 0,0 & 0 & 0,0 & 10 & 3,5 & 2 & 0,7 & 0 & 0,0 & 12 & 4,2 \\
\hline Malo & 0 & 0,0 & 5 & 1,7 & 0 & 0,0 & 43 & 15,0 & 1 & 0,3 & 49 & 17,1 \\
\hline Muy malo & 2 & 0,7 & 0 & 0,0 & 0 & 0,0 & 0 & 0,0 & 12 & 4,2 & 14 & 4,9 \\
\hline Total & 2 & 0,7 & 5 & 1,7 & 221 & 77,3 & 45 & 15,7 & 13 & 4,5 & 286 & 100 \\
\hline
\end{tabular}

Fuente. ficha de los hábitos alimentarios e IMC. 
1) Nivel de significancia

$$
<=0,01 \text { (error de la prueba) }
$$

\section{2) Estadístico de prueba y región critica}

La estadística de prueba es:

$$
X_{c a l}^{2}=\sum_{i=j}^{r} \sum_{j=i}^{c} \frac{\left(\mathrm{O}_{i j}-\varepsilon_{i j}\right)^{2}}{\varepsilon_{i j}}=\operatorname{aproximado} X_{1-\alpha(4-1)(5-1)}^{2}
$$

Para un nivel de significancia de

$$
<=0,05 \text { y (4-1) 5-1) = } 12 \text { grados de libertad }
$$

el valor crítico es $x_{1-0.05,12}^{2}=21,03$

\section{3) Cálculo y toma de decisión}

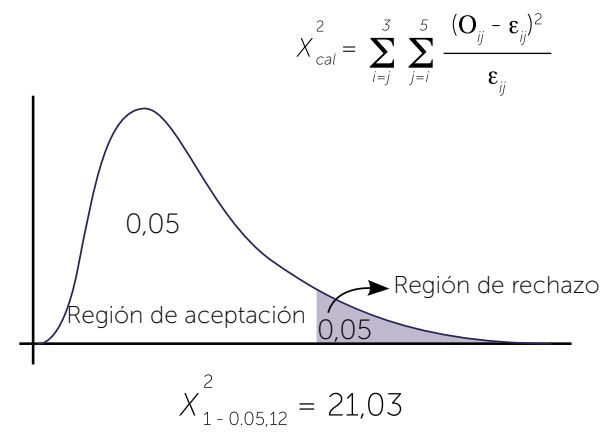

$x_{c a l}^{2}=\frac{(0-0,09)^{2}}{0,09}+\frac{(0-0,23)^{2}}{0,23}+\ldots .+\frac{(0-0,05)^{2}}{0,05}=10,7616$

A partir del cálculo precedente, se acepta la hipótesis nula $(\mathrm{Ho})$ y se rechaza la hipótesis alterna $(\mathrm{Ha})$. Por lo que se concluye que no existe relación entre el nivel socioeconómico y el estado nutricional de los escolares del sur peruano fundamentado con una probabilidad del $95 \%$ de confianza.

Por otro lado, en el presente trabajo de investigación se obtuvieron los siguientes resultados emanados según la encuesta socioeconómica:

\section{Acceso a la vivienda}

Se encontró que la mejor calidad de vivienda pertenece a estudiantes escolares con un estado nutricional normal, sobrepeso y en menor cantidad los de obesidad y de riesgo de delgadez. Las viviendas de menor calidad corresponden en estudiantes con diagnostico nutricional de normal, obesidad, riesgo de delgadez y delgadez. Además, se halló que la cocina es una pieza independiente en un 89,86\% de estudiantes escolares.

\section{Acceso a servicios}

Referente a la procedencia de agua que consumen, la mayoría de estudiantes cuentan con pileta domiciliaria y tienen diagnostico normal. Los estudiantes que obtienen por pileta publica y pozo domiciliario son de menor proporción y tienen un diagnostico normal, sobrepeso, obesidad y delgadez.

\section{Capacidad económica}

Respecto al ingreso familiar, se obtuvo que más del 50 \% tiene ingresos entre S/ 750 y S/ 1200 y los estudiantes se encuentran con diagnostico nutricional normal y una menor cantidad con sobrepeso. Los estudiantes con ingresos mayores a S/ 1200 tienen un diagnostico nutricional normal, sobrepeso y obesidad. Finalmente, los de ingresos menores de S/ 750 tienen un diagnostico nutricional normal, sobrepeso, obesidad y delgadez.

\section{Ocupación}

El padre de la mayoría de estudiantes trabaja en el sector privado y como pequeño comerciante. Por otro lado, las madres de la mayoría están subempleadas, por lo que trabajan como comerciantes informales. Entonces, los estudiantes que habitan en esas familias descritas,

\begin{tabular}{|c|c|c|c|c|c|c|c|}
\hline \multirow{2}{*}{\multicolumn{2}{|c|}{$\begin{array}{l}\text { Nivel socioeconómico } \\
\text { Delgadez }\end{array}$}} & \multicolumn{5}{|c|}{ IMC } & \multirow[b]{2}{*}{ Total } \\
\hline & & \multirow{2}{*}{$\begin{array}{c}\begin{array}{c}\text { Riesgo de } \\
\text { delgadez }\end{array} \\
0\end{array}$} & \multirow{2}{*}{$\begin{array}{c}\text { Normal } \\
0\end{array}$} & \multirow{2}{*}{$\begin{array}{c}\text { Sobrepeso } \\
10\end{array}$} & \multicolumn{2}{|l|}{ Obesidad } & \\
\hline \multirow{2}{*}{ Alto } & Observado & & & & 3 & 0 & 13 \\
\hline & Esperado & 0,09 & 0,23 & 10,05 & 2,05 & 0,59 & 13 \\
\hline \multirow{2}{*}{ Medio } & Observado & 1 & 5 & 160 & 30 & 8 & 204 \\
\hline & Esperado & 1,43 & 3,57 & 157,64 & 32,10 & 9,27 & 204 \\
\hline \multirow{2}{*}{ Bajo-superior } & Observado & 1 & 0 & 51 & 11 & 5 & 68 \\
\hline & Esperado & 0,48 & 1,19 & 52,55 & 10,70 & 3,09 & 68 \\
\hline \multirow{2}{*}{ Bajo-inferior } & Observado & 0 & 0 & 0 & 1 & 0 & 1 \\
\hline & Esperado & 0,01 & 0,02 & 0,77 & 0,16 & 0,05 & 1 \\
\hline Total & Recuento & 2 & 5 & 221 & 45 & 13 & 286 \\
\hline
\end{tabular}

Tabla 5

Contingencia en relación del nivel socioeconómico e IMC

Fuente: ficha del nivel socioeconómico e IMC. 
presentan diagnostico nutricional normal y en menor cantidad sobrepeso y riesgo de delgadez.

Los resultados obtenidos en el presente estudio muestran que la mayoría de los estudiantes con estado nutricional normal pertenecen a los niveles socioeconómicos medio y bajo superior; lo cual muestra que los alumnos en su mayoría cuentan con una adecuada calidad de vivienda, economía estable y servicios básicos, además del acceso a alimentos. Sin embargo, los estudiantes con estado nutricional con riesgo de delgadez pertenecen al nivel socioeconómico medio y los estudiantes con estado nutricional con delgadez pertenecen a los niveles socioeconómicos medio y bajo superior, lo cual no se ha resuelto hasta la actualidad.

\section{Comprobación de la segunda hipótesis específica de los hábitos alimentarios}

Ha: Existe relación entre los hábitos alimentarios y el estado nutricional de escolares del sur peruano.

Ho: No existe relación entre en el nivel socioeconómico y el estado nutricional de escolares del sur peruano.

\section{Nivel de significancia}

$$
<=0,05 \text { (error de la prueba) }
$$

\section{Estadístico de prueba y región critica}

La estadística de prueba es:

$$
X_{c a l}^{2}=\sum_{i=j}^{r} \sum_{j=i}^{c} \frac{\left(\mathrm{O}_{i j}-\varepsilon_{i j}\right)^{2}}{\varepsilon_{i j}}=\text { aproximado } X_{1-\alpha(5-1)(5-1)}^{2}
$$

Para un nivel de significancia de

$$
<=0,05 \text { y }(5-1) 5-1)=16 \text { grados de libertad }
$$

el valor crítico es $x_{1-0.05,16}^{2}=26,30$

3) Cálculo y toma de decisión

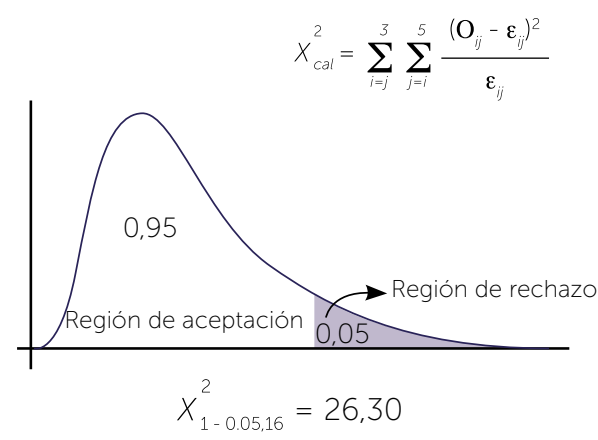

$$
x_{c a l}^{2}=\frac{(0-0,03)^{2}}{0,01}+\frac{(0-0,07)^{2}}{0,07}+\ldots .+\frac{(12-0,64)^{2}}{0,64}=536,5621
$$

Del análisis estadístico anterior, la prueba de chicalculada muestra que la chi de tabla tiene un nivel de significancia de 0,05 y 16 grados de libertad. Por tanto, se rechaza la hipótesis nula $(\mathrm{Ho})$ y se acepta la hipótesis alterna ( $\mathrm{Ha}$ ), concluyendo de esa manera que existe relación entre los hábitos alimentarios y el estado

\begin{tabular}{|c|c|c|c|c|c|c|c|}
\hline \multirow{2}{*}{\multicolumn{2}{|c|}{$\begin{array}{l}\text { Hábitos alimentarios } \\
\text { Delgadez }\end{array}$}} & \multicolumn{5}{|c|}{ IMC } & \multirow{2}{*}{ Total } \\
\hline & & \multirow{2}{*}{$\begin{array}{c}\begin{array}{c}\text { Riesgo de } \\
\text { delgadez }\end{array} \\
0\end{array}$} & \multirow{2}{*}{$\frac{\text { Normal }}{0}$} & \multirow{2}{*}{$\frac{\text { Sobrepeso }}{4}$} & \multicolumn{2}{|l|}{ Obesidad } & \\
\hline \multirow{2}{*}{ Muy bueno } & Recuento & & & & 0 & 0 & 4 \\
\hline & Frecuencia esperada & 0,03 & 0,07 & 3,09 & 0,63 & 0,18 & 4 \\
\hline \multirow{2}{*}{ Bueno } & Recuento & 0 & 0 & 207 & 0 & 0 & 207 \\
\hline & Frecuencia esperada & 1,45 & 3,62 & 159,95 & 32,57 & 9,41 & 207 \\
\hline \multirow{2}{*}{ Regular } & Recuento & 0 & 0 & 10 & 2 & 0 & 12 \\
\hline & Frecuencia esperada & 0,08 & 0,21 & 9,27 & 1,89 & 0,55 & 12 \\
\hline \multirow{2}{*}{ Malo } & Recuento & 0 & 5 & 0 & 43 & 1 & 49 \\
\hline & Frecuencia esperada & 0,34 & 0,86 & 37,86 & 7,71 & 2,23 & 49 \\
\hline \multirow{2}{*}{ Muy malo } & Recuento & 2 & 0 & 0 & 0 & 12 & 14 \\
\hline & Frecuencia esperada & 0,10 & 0,24 & 10,82 & 2,20 & 0,64 & 14 \\
\hline Total & Recuento & 2 & 5 & 221 & 45 & 13 & 286 \\
\hline
\end{tabular}
nutricional de estudiantes del sur del Perú.

Tabla 6

Contingencia en relación de los hábitos alimentarios e IMC

Fuente. ficha de los hábitos alimentarios e IMC. 


\section{DISCUSIÓN}

Hablar del estado nutricional en la esfera educativa nacional implica la permeabilidad de ciertos patrones en la alimentación de uno de los actores educativos: los estudiantes. Mientras que los otros actores educativos: padres de familia, no han podido regular la alimentación de sus propios hijos debido a muchos factores como la carencia de tiempo que se supone que deberían asignarles

Existen estudios realizados en el sur peruano que muestran un estado nutricional de 295 estudiantes de 12 a 16 años, en el cual se encontró que el 3,29 \% tenía un diagnóstico de delgadez, el 80,56\% estaba en los parámetros normales, el 13,42 \% estaba con sobrepeso y el 2,74 \% presentaba obesidad (Begazo, 2008). Bajo ese paralelismo, se observa que en el estudio actual la obesidad ha incrementado levemente. A pesar de que Shamah-Levy et al. (2018) argumentan que la prevalencia global de sobrepeso y obesidad en niñosadolescentes se ha estabilizado, en el sur peruano sigue en ascenso.

Haciendo un contrataste que implica a las instituciones estudiadas, se materializa la tendencia a la ingesta calórica de grasas saturadas y otras comidas nada saludables para el menor en latencia, lo que induce a una atmósfera propicia para el desarrollo de problemas nutricionales y otros componentes en perjuicio de los estudiantes (Azañedo-Vilchez, 2017). Por otro lado, la mala alimentación trae consigo la desnutrición crónica como otro factor de riesgo para la salud, la sobrevivencia (Cuevas-Nasu et al., 2018) y el buen aprendizaje de los adolescentes.

Tratar de comprender cómo afecta la economía en la alimentación de los estudiantes, requiere de una tipología que tenga en cuenta la influencia del tiempo explicado en tres momentos: primero, la pobreza persistente a la cual están sometidos los hombres del altiplano; segundo, la pobreza temporal que tiene una directa relación con la exclusión y, tercero, se presenta la pobreza recurrente como resultado del mal salario que mantienen las familias (Huanca-Arohuanca, 2019; 2020b; 2020c; 2021a; 2021b; Tejero, 2018). A pesar de que el estudio arroja como resultado la relación inexistente entre la variable socioeconómico con el estado nutricional, muchas veces el tema económico es el que crea la variable pobreza.

En tal sentido, el tema socioeconómico está en directa vinculación con la categoría de pobreza, tal como D'Amico (2015) manifiesta que este tema se ha discutido desde finales del siglo XX a escala planetaria, porque su tratamiento se hacía cada vez más notorio por las mismas complejidades y ciertas irregularidades multidimensionales que manifestaba el hambre. Entonces, hasta la actualidad, lo único que esas perspectivas han hecho es consolidar el proceso de empobrecimiento hacia las poblaciones más vulnerables como el Perú.

A inicios de la segunda década del siglo XXI, todavía persiste la diferencia de clase que explica muy bien los hábitos alimentarios manejados como rutina por los estudiantes del sur peruano. Por tanto, aún se habla de comidas de ricos y comidas de pobres en Gracia (como se citó en Díaz y García, 2018); lo cual tiene un significado potente en el engranaje educativo que todavía no se ha superado. Hablar de alimentos para ricos y pobres es continuo en los colegios, porque existen pequeños grupos de estudiantes que comen otras cosas a diferencia de la mayoría carente. Empero, lo descrito puede no tener relevancia a la hora de seleccionar los alimentos no tan nutritivos para la salud, ya que, generalmente, el que trae más dinero elige comidas poco nutritivas.

Por otra parte, se inserta nuevamente la idea de la diferencia de clases que aún se mantiene en el sur del Perú. Fuera de cualquier duda, el tema ha sido trabajado desde varios enfoques y en muchas investigaciones, pero en el campo alimentario se carece todavía de una explicación medular. Por ello, se debe indicar que la diferencia de clases es una tipificación que ha puesto en dos variantes a los estudiantes de corte urbano y los procedentes de la marginalidad. Es decir, los estudiantes de la zona urbana manifiestan tener una mejor propina, que es lo mismo que el dinero para gastar en el recreo; lo que además llevará a que la alimentación sea más descontrolada, mientras los carentes de economía son regulados por el mismo rol monetario.

La explicación anterior puede ser coherente hasta cierto punto, sin embargo, el estudio indica que lo monetario no implica que los que tienen más puedan tener más inclinaciones a la obesidad. Entonces, el problema de fondo está en los proveedores que incitan a comidas sin ningún tipo de valor nutritivo. Por lo que las instituciones educativas deberán ensayar otro tipo de estrategias para regular lo que se vende dentro y fuera de sus espacios conocidos como tiendas escolares, motivados por ciertas empresas mundiales de comida.

En ese sentido, el estudio referente a los hábitos alimentarios en Perú es un tema relevante para mostrar los efectos de la mundialización que rompe los esquemas construidos en la vida cotidiana (Bertran, 2017). Sabiendo que después de la exposición de comidas sin ningún valor nutritivo por el fenómeno en referencia, la comunidad estudiantil se ha vuelto dependiente a la comida ligera. Por eso, el desafío está 
en replantear nuevas políticas desde el Estado para que puedan flagelar la inserción de esos alimentos perjudiciales para la salud de sus habitantes escolares.

La investigación tuvo limitaciones, en el sentido de que no encontró fuentes teóricas actuales similares al tema en tratamiento, lo cual dificultó relativamente la construcción del estado del arte; por lo tanto, se debe señalar que el estudio servirá de base para la construcción de otras investigaciones altiplánicas.

\section{CONCLUSIONES}

El tema nutricional es un asunto poco trabajado en los espacios australes del Perú, sobre todo cuando los actores son estudiantes de los diferentes espacios territoriales, ya sea urbano o netamente rural. En ese sentido, el estudio llega a dos conclusiones trascendentales que merecen un tratamiento minucioso en su variabilidad tanto socioeconómica o habitualidad alimentaria.

La primera variable sustenta que no existe relación entre el nivel socioeconómico y el estado nutricional de los escolares del sur peruano; todo ello, fundamentado con una probabilidad del $95 \%$ de confianza. A pesar de que la región altiplánica enfrenta una serie de precariedades, el asunto monetario no resulta determinante en su inserción de la calidad del estado nutricional.

La segunda variable rechaza la hipótesis nula y acepta la hipótesis alterna de manera tácita, concluyendo por lo tanto que existe una relación entre los hábitos alimentarios y el estado nutricional de estudiantes con un nivel de significancia del 0,05. Situación que resulta preocupante para los estamentos educativos y los actores educativos encargados de velar por la salud de los estudiantes.

\section{REFERENCIAS}

Arnaud, R., López, M., y Mataix, J. (2005). Entorno social y desnutrición en niños de 1 a 4 años de comunidades indígenas de México. Rev Esp de Nutr Comunitaria, 11(3), 128-134. https://www.renc.es/imagenes/auxiliar/files/entorno_20social_l_1155031268391.pdf

Azañedo-Vilchez, D. E. (2017). Guías alimentarias: una opción para seguir enfrentando los problemas nutricionales en el Perú. Salud Pública de México, 59(3), 215217. https://doi.org/10.21144/8046

Begazo, H. (2008). Influencia de hábitos alimentarios en la prevalencia de sobrepeso y obesidad en escolares adolescentes de instituciones educativas de la ciudad de Puno. Universidad Nacional del Altiplano.

Bertran, M. (2017). Anales de Antropología Domesticar la globalización: alimentación y cultura en la urbaniza- ción de una zona rural en México. Anales de Antropología, 51(2), 123-130. https://doi.org/10.1016/j. antro.2017.05.003

Bobroff, L. B. (2012). Vida Saludable: determine su salud nutricional. University of Florida. https://ufdcimages. uflib.ufl.edu/IR/00/00/08/92/00001/FY132600.pdf

Burstein-Roda, T. (2018). Reflexiones sobre la gestión de los recursos hídricos y la salud pública en el Perú. Rev Peru Med Exp Salud Publica, 35(2), 297-303. https://doi.org/10.17843/rpmesp.2018.352.3641.297

Cuevas-Nasu, L., Shamah-Levy, T., Hernández-Cordero, S. L., González-Castell, L. D., Gómez-Humarán, I. M., Ávila-Arcos, M. A. y Rivera-Dommarco, J. A. (2018). Tendencias de la mala nutrición en menores de cinco años en México, 1988-2016: análisis de cinco encuestas nacionales. Salud Pública de México, 60(3), 283-290. https://doi.org/10.21149/8846

D'Amico, V. (2015). De la pobreza a la desigualdad. Discursos internacionales, efectos nacionales. Latinoamérica. Revista de Estudios Latinoamericanos, 61, 237-264. https://doi.org/10.1016/j.larev.2015.12.010

Díaz, C. y García, I. (2018). Homogeneidad y fragmentación en los hábitos alimentarios de los españoles. Una respuesta a través del análisis de los horarios, las relaciones y las normas alimentarias. Revista Internacional de Sociología, 76(3), e102. https://doi. org/10.3989/ris.2018.76.3.17.360

Huanca-Arohuanca, J. W. (2019). El despertar de una nueva era: colonización y camino hacia la revolución. Revista Revoluciones, 1(1), 1-3. http://revistarevoluciones.com/index.php/rr/article/view/1/2

Huanca-Arohuanca, J. W. (2020a). Caleidoscopio social al Covid-19: pánico y desesperación en tiempos de aislamiento. Revista Universidad y Sociedad, 12(6), 226-231. https://rus.ucf.edu.cu/index.php/rus/article/view/1836

Huanca-Arohuanca, J. W. (2020b). Contrahegemonía y la lucha por la educación en el sur del Perú. Editorial Académica Española.

Huanca-Arohuanca, J. W. (2020c). Retropías y distopías de la educación en Puno. Revista Helios, 4(1), 270-271. https://doi.org/10.22497/Helios.41.4116

Huanca-Arohuanca, J. W. (2021a). Narrativas de guerra y resistencia: participación de la mujer austral del Perú en la Guerra del Pacífico. Encuentros. Revista de Ciencias Humanas, Teoría Social y Pensamiento Crítico, 13, 50-59. https://doi.org/http://doi. org/10.5281/zenodo.4395218

Huanca-Arohuanca, J. W. (2021b). Un fantasma recorre el Perú del Bicentenario: lecciones y voluntad popular en las elecciones de 2021. Revista Revoluciones, 3(3), 1-4. https://doi.org/10.35622/j.rr.2021.03.001

Huanca-Arohuanca, J. W., Butrón, S. B., Supo, L. A. y Supo, F. (2020). Evaluación y monitoreo de la calidad ambiental del agua en el proyecto sistema de riego Canal N, provincia de Melgar - Puno, Perú. Ciencia y Desarrollo, 19(1), 88-96. http://revistas.unjbg.edu. pe/index.php/cyd/article/view/936/1062

Huanca-Arohuanca, J. W. y Geldrech, P. (2020). Planificación educativa y gestión pedagógica-estratégica-opera- 
cional en las instituciones del nivel inicial en el sur del Perú. Revista Conrado, 16(76), 369-376. https:// conrado.ucf.edu.cu/index.php/conrado/article/ view/1497

Huanca-Arohuanca, J. W. y Núñez, L. (2020). Estimaciones y contrastes de la pandemia en Perú y en el contexto mundial. Educare Et Comunicare. Revista Cientifica de La Fcultad de Humanidades, 8(2), 10-20. https://doi.org/10.35383/educare.v8i2.440

Huanca-Arohuanca, J. W. y Pilco, N. (2021). Acciones revolucionarias en Ámérica Latina: Puno y el Alto Perú durante el proceso de independencia (1809-1825). Chakiñan. Revista de Ciencias Sociales y Humanidades, 14. https://doi.org/10.1590/SciELOPreprints.1364

Huanca-Arohuanca, J. W., Supo-Condori, F., Sucari, R. y Supo, L. A. (2020). El problema social de la educación virtual universitaria en tiempos de pandemia, Perú. Revista Innovaciones Educativas, 22, 115-128. https://doi.org/10.22458/ie.v22iespecial.3218

Instituto Nacional de Estadística e Informática. (2007). Censo de población y vivienda. http://censos.inei.gob.pe/ cpv2007/tabulados/

Miguel-Aguilar, C. F., Rodríguez-Bolaños, R. de los Á., Caballero, M., Arillo-Santillán, E. y Reynales-Shigematsu, L. M. (2017). Fumar entre adolescentes: análisis cuantitativo y cualitativo de factores psicosociales asociados con la decisión de fumar en escolares mexicanos. Salud Publica de México, 59, S63-S72. https://doi.org/10.21149/7835

Morales-Ruán, M. del C., Shamah-Levy, T., Mundo-Rosas, V., Cuevas-Nasu, L., Lozada-Tequeanes, A. L., y Romero-Martínez, M. (2018). Evolución de los programas de ayuda alimentaria en México a través de información de la Ensanut MC 2016. Salud Pública de México, 60(3), 319-327. https://doi.org/10.21149/8818

Morgante, M. G. y Remorini, C. (2018). Estudio etnográfico de las relaciones intergeneracionales en el cuidado de la salud a escala doméstica durante las etapas pre y postnatal (Molinos, Salta, Argentina). Apuntes, 45(83), 37-65. https://doi.org/10.21678/apuntes.83.909

Navarro-Aburto, B., Díaz-Bustos, E., Muñoz-Navarro, S. y Pérez-Jiménez, J. (2017). Condición física y su vinculación con el rendimiento académico en estu- diantes de Chile. Revista Latinoamericana de Ciencias Sociales, Niñez y Juventud, 15(1), 309-325. https://doi.org/10.11600/1692715x.1511902032016

Shamah-Levy, T., Cuevas-Nasu, L., Gaona-Pineda, E. B., Gómez-Acosta, L. M., Morales-Ruán, M. del C., Hernández-Ávila, M. y Rivera-Dommarco, J. Á. (2018). Sobrepeso y obesidad en niños y adolescentes en México, actualización de la Encuesta Nacional de Salud y Nutrición de Medio Camino 2016. Salud Pública de México, 60(3), 244-253. https://doi. org/10.21149/8815

Tejero, A. (2018). Pobreza laboral en España. Un análisis dinámico. Revista Internacional de Sociología, 76(2). https://doi.org/10.3989/ris.2018.76.2.16.54

Uribe-Carvajal, R., Jiménez-Aguilar, A., Morales-Ruan, M. del C., Salazar-Coronel, A. A. y Shamah-Levy, T. (2018). Percepción del peso corporal y de la probabilidad de desarrollar obesidad en adultos mexicanos. Salud Pública de México, 60(3), 254-262. https://doi. org/10.21149/8822

\section{Contribución de los autores}

LLT: introducción, análisis del problema y revisión del artículo científico.

PGS: análisis de resultados de la investigación, revisión final del artículo y actualización de Mendeley.

YQS: diseño metodológico, revisión final del artículo y levantamiento de observaciones.

HM-C: recolección de datos y revisión del artículo científico.

JWH-A: idea original del proyecto, planeación del estudio y redacción del artículo científico.

\section{Fuentes de financiamiento}

La investigación fue realizada con recursos propios.

\section{Conflictos de interés}

El autor declara no tener conflicto de interés.

\section{Correspondencia}

Jesús Wiliam Huanca-Arohuanca

Av, Panmericana, Perú.

C.P. 04001

Cel.: 974323230

Email: jhuancaar@unsa.edu.pe 